\title{
Reproductive biology of the yellow snapper, Lutjanus argentiventris (Pisces, Lutjanidae), from the Mexican central Pacific
}

\section{Biología reproductiva del pargo alazán, Lutjanus argentiventris (Pisces, Lutjanidae), en el Pacífico central mexicano}

\author{
Gabriela Lucano-Ramírez, Salvador Ruiz-Ramírez, Gaspar González-Sansón", \\ Bertha Patricia Ceballos-Vázquez² \\ ${ }^{1}$ Universidad de Guadalajara, Departamento de Estudios para el Desarrollo Sustentable de Zonas Costeras, \\ V. Gómez Farías 82, San Patricio-Melaque, Jalisco, México. \\ ${ }^{2}$ Instituto Politécnico Nacional-Centro Interdisciplinario de Ciencias Marinas, Av. Instituto Politécnico \\ Nacional s/n, Col. Playa Palo de Santa Rita, AP 592, La Paz 23096, BCS, México \\ * Corresponding author. E-mail: lucanorg@costera.melaque.udg.mx
}

\begin{abstract}
The yellow snapper, Lutjanus argentiventris, is a commercial species along the Pacific coast, yet few studies have been carried out on its reproductive biology. Monthly samplings of the artisanal fishery off the coast of Jalisco, Mexico, were made from 1998 to 2008. Mean total length and weight of analyzed specimens were $32.6 \mathrm{~cm}( \pm 0.13)$ and $567.3 \mathrm{~g}( \pm 9.15)$, respectively. A total of 945 females $(50.3 \%)$ and 932 males (49.7\%) were collected; the overall sex ratio was 1:0.99. The highest percentages of mature fish were obtained between July and October. The highest gonadosomatic index values (1.46 to 0.81$)$ and highest oocyte diameters were also obtained during that period. For this reason the period from July to October is considered the breeding season of L. argentiventris. This species presented asynchronous ovarian development and seven stages of oocyte development were identified. The testis showed lobular development and in the ripe stage, sperm were observed in the center of the lobes and in the main duct. Mean size at sexual maturity $\left(L_{50}\right)$ was $31.6 \mathrm{~cm}$ for females and $31.4 \mathrm{~cm}$ for males, and there was no significant variation in $L_{50}$ among years. Due to the long period of study, this information could be used to establish measures for the proper exploitation of the species.
\end{abstract}

Key words: Lutjanidae, sex ratio, maturity stages, gonadosomatic index, length at maturity.

RESUMEN. El pargo alazán Lutjanus argentiventris, es una especie que se comercializa a lo largo de las costas del Pacífico. Sin embargo, pocos estudios se han enfocado en conocer las características biológicas de la especie. Se realizaron muestreos mensuales de los organismos capturados en la pesca artesanal de 1998 a 2008 frente a la costa de Jalisco, México. La longitud total promedio de los ejemplares analizados fue de $32.6 \mathrm{~cm}( \pm 0.13)$ y el peso total promedio fue de $567.3 \mathrm{~g}( \pm 9.15)$. Se recolectaron un total de 945 hembras $(50.3 \%)$ y $932 \mathrm{machos}$ (49.7\%); la proporción de sexos total fue de 1:0.99. Los mayores porcentajes de peces maduros se encontraron de julio a octubre. Durante el mismo periodo, se registraron los valores del índice gonadosomático más altos (1.46 a 0.81$)$ y los mayores diámetros de ovocitos. Por esta razón, el periodo de julio a octubre se considera la temporada reproductiva de L. argentiventris. El ovario de esta especie presentó un desarrollo asincrónico, y se identificaron siete fases de desarrollo del ovocito. El testículo presentó un desarrollo del tipo lobular, y en el estadio maduro los espermatozoides se localizaron en el centro de los lóbulos y en el conducto principal. La talla promedio de madurez $\left(L_{50}\right)$ fue de $31.6 \mathrm{~cm}$ para las hembras y $31.4 \mathrm{~cm}$ para los machos, y no se observó una variación significativa en la $L_{50}$ entre los años muestreados. Debido al largo periodo de este estudio, esta informacion podría utilizarse para establecer medidas para la explotación propia de esta especie.

Palabras clave: Lutjanidae, proporción sexual, estadios de madurez, índice gonadosomático, talla de madurez.

\section{INTRODUCTION}

The yellow snapper, Lutjanus argentiventris (Peters 1869), inhabits sandy bottoms and is distributed from southern California to Peru. Juvenile yellow snapper form schools but the adults are solitary (Amezcua-Linares 1996, Allen and Robertson 1998). In the Mexican tropical Pacific, this species is caught by gillnets, hook and line, harpoon, and trawl nets. In this region, L. argentiventris is one of the 10 most important species (Rojo-Vázquez et al. 2008). The annual landings of the artisanal fleet have oscillated between 0.5 and $1.5 \mathrm{t}$

\section{INTRODUCCIÓN}

El pargo alazán, Lutjanus argentiventris (Peters 1869), habita en fondos arenosos y se distribuye desde el sur de California hasta Perú. Los juveniles de esta especie forman grupos, pero los adultos son solitarios (Amezcua-Linares 1996, Allen y Robertson 1998). En el Pacífico tropical mexicano, esta especie se captura con redes agalleras, anzuelos, línea, arpón y redes de arrastre. En esta región, L. argentiventris es una de las 10 especies de mayor importancia (Rojo-Vázquez et al. 2008). Su captura comercial 
(Espino-Barr et al. 2004); however, local fishermen have repeatedly mentioned that the number of yellow snapper landed has declined in recent years.

Despite the economic importance and abundance of L. argentiventris, its biological characteristics have been poorly studied (Cruz-Romero et al. 1996, Rojas et al. 2004, Piñón et al. 2009). Moreover, there are no studies on its ecology and the effect of fishing on the species, even though such information is necessary to develop effective programs for the protection and management of the resource. Spawning biomass is one of the reference points for assessing the status of an exploited stock and establishing future catch levels (Vitale et al. 2006).

In view of the scant information available on the reproductive biology of $L$. argentiventris, the objective of this paper is to analyze the sex ratio, breeding season, and size at maturity of this species off the Pacific coast of central Mexico. The microscopic characteristics of the ovary and testis are also described.

\section{MATERIALS AND METHODS}

On five days of every month between January 1998 and December 2008 (except from February to October 2001), yellow snapper specimens were obtained from the commercial landings of artisanal fishermen operating gillnets of different mesh sizes in Navidad Bay, Tenacatita Bay, and Chamela Bay on the coast of the Mexican state of Jalisco. The total length (TL, $\pm 0.1 \mathrm{~cm}$ ) and total weight (TW, $\pm 0.1 \mathrm{~g}$ ) of each specimen were recorded. Sex and maturity stages were identified by direct observation of the gonads. Gonads were removed, weighed ( $\mathrm{GW}, \pm 0.01 \mathrm{~g}$ ), and fixed in $10 \%$ neutral formol for subsequent histological analysis. The data from all years were pooled for the analysis.

Sex ratio was calculated for each year and for the total sample size. A chi-square $\left(\chi^{2}\right)$ goodness-of-fit test incorporating Yates' correction for continuity (Zar 2010) was performed to compare and determine if the sex ratio differed from the expected $1: 1$.

To determine the breeding season, three complementary methods were used. The first consisted of calculating the gonadosomatic index $(\mathrm{GSI}=[\mathrm{GW} /(\mathrm{TW}-\mathrm{GW})] \times 100)$. This index is widely used as an indicator of the reproductive cycle, and assumes that the maximum mean values indicate gonadal maturity (Sánchez-Cárdenas et al. 2007). The second method consisted of assessing gonadal maturation based on a morphochromatic scale of four development stages (immature, developing, ripe, and spent) according to the criteria proposed by Everson et al. (1989), considering the highest percentages of organisms in stages of greater maturation per month to determine the breeding season. The third method consisted of analyzing the monthly variation in oocyte diameter, assuming that a higher diameter corresponds to a greater degree of maturation of the ovary. For this, 10 oocytes randomly selected from the severn stages of development ribereña ha oscilado de 0.5 a 1.5 t por año (Espino-Barr et al. 2004); sin embargo, los pescadores locales han reportado reiteradamente que el número de pargos alazán capturados ha disminuido en los últimos años.

A pesar de la importancia económica y la abundancia de L. argentiventris, los estudios sobre su biología son escasos (Cruz-Romero et al. 1996, Rojas et al. 2004, Piñón et al. 2009). Además, no existen estudios sobre su ecología ni el efecto de las pesquerías sobre esta especie, a pesar de que dichos estudios son necesarios para desarrollar programas efectivos para la protección y manejo del recurso. La biomasa desovante es uno de los puntos de referencia para evaluar el estado de una población explotada y para establecer futuros niveles de captura (Vitale et al. 2006).

Debido a la importancia de L. argentiventris y a la poca información existente sobre la biología reproductiva, el objetivo de este trabajo fue analizar la proporción sexual, época reproductiva y talla de madurez de esta especie en las costas de Jalisco. También se describen las características microscópicas del ovario y del testículo.

\section{MATERIALES Y MÉTODOS}

De enero de 1998 a diciembre de 2008 (excepto de febrero a octubre de 2001), durante cinco días al mes, se obtuvieron ejemplares de pargo alazán de la captura comercial realizada por pescadores ribereños con redes agalleras de diferente luz de malla en las bahías de Navidad, Tenacatita y Chamela, ubicadas en la costa de Jalisco, México. Se registró la longitud total ( $\mathrm{LT}, \pm 0.1 \mathrm{~cm}$ ) y el peso total (PT, $\pm 0.1 \mathrm{~g}$ ) de cada ejemplar. El sexo y el grado de madurez se identificaron mediante la observación directa de las gónadas. Las gónadas fueron extraídas, pesadas (PG, $\pm 0.01 \mathrm{~g}$ ) y fijadas en formol neutro al $10 \%$ para su posterior análisis histológico. Para los análisis, se agrupó la información de todos los años.

La proporción sexual fue calculada para cada año y para el tamaño de muestra total. Se utilizó la prueba de bondad de ajuste chi cuadrada $\left(\chi^{2}\right)$ con corrección para continuidad de Yates (Zar 2010) para comparar y determinar si la proporción de sexos difería de la esperada 1:1.

Para conocer la temporada reproductiva, se aplicaron tres métodos complementarios. El primero consistió en el cálculo del índice gonadosomático $(\mathrm{IGS}=[\mathrm{PG} /(\mathrm{PT}-\mathrm{PG})] \times 100)$; este índice es ampliamente usado como indicador del periodo reproductivo, bajo el supuesto de que los valores promedio máximos indican madurez gonádica (Sánchez-Cárdenas et al. 2007). El segundo método consistió en evaluar la maduración gonadal con base en la escala morfocromática de cuatro estadios de desarrollo gonádico (inmaduro, en desarrollo, maduro y desovado) de acuerdo con los criterios de Everson et al. (1989), tomando en cuenta los mayores porcentajes de organismos en estadios de mayor maduración por mes para ubicar la temporada reproductiva. El tercer método consistió en analizar la variación mensual del diámetro de los ovocitos, suponiendo que un mayor diámetro corresponde a un mayor grado 
were measured (largest and smallest diameter of the oocyte nucleus) (West 1990).

To describe the internal structure of the gonad, histological sections were taken from ovaries and testes: gonads were dehydrated in ethyl alcohol, embedded in paraplast, and sections of 6-8 $\mu \mathrm{m}$ thickness were stained with hematoxylin and eosin. The sections were observed under a Zeiss Axiostar Plus microscope. Oocyte development stages were classified according to Yamamoto and Yamazaki (1961), Cerisola (1984), and Lucano-Ramírez et al. (2001b). The internal structure of the testis was described according to Hyder (1969) and Lucano-Ramírez et al. (2001a).

One-way analysis of variance (ANOVA) was performed to evaluate the annual changes in TL and TW. When the ANOVA showed significant differences $(P \leq 0.05)$, the Newman-Keuls multiple range test was applied using Statistica (v7.0). The condition factor $\left(\mathrm{CF}=\left[\mathrm{TW} / \mathrm{TL}^{3.01}\right] \times 100\right)$ was obtained as an indirect indicator of the physiological condition of the population. The Spearman rank correlation test $\left(r_{\mathrm{s}}\right)$ was used to evaluate the monthly relation between $\mathrm{CF}$ and GSI, and between GSI and mean oocyte diameter.

The length at which $50 \%$ of the individuals are sexually mature $\left(L_{50}\right)$ was estimated by fitting the number of mature and immature organisms in each size class (4-cm intervals) to the logistic model $\left(L_{50}=1 /\left[1+e^{(b \mathrm{TL}+a)}\right]\right)$. Fitting was done by calculating the non-linear approximation for each sex by maximum likelihood with a bionomial distribution. The point value for each sex was obtained based on macroscopic criteria (Everson et al. 1989). The temporal trend of maturity size was determined by regression analysis of the survey years and $L_{50}$.

\section{RESULTS}

\section{Size composition and sex ratio}

A total of 1882 yellow snapper were examined. Five of them, which ranged from 20.8 to $22.3 \mathrm{~cm}$ TL, could not be sexed. Females ranged from 23.3 to $62.0 \mathrm{~cm}$ TL, with a mean of $33.3 \mathrm{~cm}( \pm 0.20$, standard error), and from 172 to $3450 \mathrm{~g}$ TW, with a mean of $611.8 \mathrm{~g}( \pm 14.48)$. Males ranged from 22.7 to $60.0 \mathrm{~cm} \mathrm{TL}$, with a mean of $32.0 \mathrm{~cm}( \pm 0.17)$, and from 172 to $3450 \mathrm{~g}$ TW, with a mean of $523.8 \mathrm{~g}( \pm 10.98)$ (fig. 1). The interannual variations of different morphometric variables are shown in table 1 . Mean lengths, recorded in the different years of the survey, ranged from 29.8 to $37.7 \mathrm{~cm}$ TL. In 2008, both females and males were significantly $(P<$ 0.05 , ANOVA) larger ( 37.7 and $34.5 \mathrm{~cm}$ TL, respectively) and heavier (945.7 and $661.5 \mathrm{~g}$ TW, respectively) relative to the other years. For females, the lowest mean size $(30.9 \mathrm{~cm}$ $\mathrm{TL})$ and weight (455.8 g TW), though not significant $(P>$ 0.05 , ANOVA), were recorded in 2001. Males were significantly $(P<0.05$, ANOVA) smaller $(29.8 \mathrm{~cm} \mathrm{TL}, 424.4 \mathrm{~g}$ TW) in 2000 (table 1). de maduración del ovario; para esto, se midieron al azar 10 ovocitos de cada fase de desarrollo (diámetro mayor y menor de los ovocitos que presentaban núcleo) (West 1990).

Para describir la estructura interna de las gónadas, se hicieron cortes histológicos de ovarios y testículos. El proceso histológico consistió en la deshidratación con alcohol etílico, inclusión en paraplast, obtención de cortes de 6-8 $\mu \mathrm{m}$ y tinción con hematoxilina-eosina de las gónadas. $\mathrm{La}$ observación de los cortes se realizó con un microscopio Axiostar Plus (Zeiss). Los diferentes tipos de ovocitos fueron clasificados de acuerdo con Yamamoto y Yamazaki (1961), Cerisola (1984) y Lucano-Ramírez et al. (2001b). La estructura interna del testículo fue descrita de acuerdo con Hyder (1969) y Lucano-Ramírez et al. (2001a).

Los cambios anuales en LT y PT se evaluaron mediante el análisis de varianza de una vía (ANDEVA). Cuando los ANDEVA mostraron diferencia significativa $(P \leq 0.05)$, se aplicó la prueba de rangos múltiples de Newman-Keuls usando el programa Statistica (v7.0). Se obtuvo el factor de condición $\left(\mathrm{FC}=\left[\mathrm{PT}^{2} \mathrm{LT}^{3.01}\right] \times 100\right)$ como indicador indirecto del bienestar fisiológico de la población. Se evaluó la relación mensual entre el FC y el IGS, y entre el IGS y el diámetro promedio de los ovocitos mediante la prueba no paramétrica de correlación por rangos de Spearman $\left(r_{\mathrm{s}}\right)$.

Se estimó la longitud a la cual el $50 \%$ de los individuos ha alcanzado la madurez sexual $\left(L_{50}\right)$ ajustando el número de organismos maduros e inmaduros en cada clase de talla (intervalos de $4 \mathrm{~cm})$ al modelo logístico $\left(L_{50}=1 /\right.$ $\left.\left[1+e^{(b \mathrm{LT}+a)}\right]\right)$. El ajuste se hizo mediante la aproximación no lineal para cada sexo por máxima verosimilitud con distribución binomial. El valor puntual para cada sexo se obtuvo con base en los criterios macroscópicos (Everson et al. 1989). Se analizó la tendencia temporal de la talla de madurez mediante un análisis de regresión entre los años de muestreo y $L_{50}$.

\section{Resultados}

\section{Composición por tallas y proporción sexual}

En total se recolectaron 1882 ejemplares de pargo alazán. A cinco de estos ejemplares, que midieron entre 20.8 y $22.3 \mathrm{~cm} \mathrm{LT}$, no se les determinó el sexo. La LT de las hembras varió de 23.3 a $62.0 \mathrm{~cm}$, con un promedio de $33.3 \mathrm{~cm}$ ( \pm 0.20 , error estándar), y la de los machos varió de 22.7 a $60.0 \mathrm{~cm}$, con un promedio de $32.0 \mathrm{~cm}( \pm 0.17)$ (fig. 1). El PT de las hembras varió de 172 a $3450 \mathrm{~g}$, con un promedio de $611.8 \mathrm{~g}$ ( \pm 14.48$)$, y el de los machos varió de 172 a $3450 \mathrm{~g}$, con promedio de $523.8 \mathrm{~g}( \pm 10.98)$. En la tabla 1 se presentan las variaciones interanuales de diferentes variables morfométricas. Las longitudes promedio, registradas en los distintos años de muestreo, fluctuaron de 29.8 a $37.7 \mathrm{~cm} \mathrm{LT}$. En 2008, tanto las hembras como los machos fueron significativamente $(P<0.05$, ANDEVA) más grandes $(37.7 \mathrm{y}$ $34.5 \mathrm{~cm} \mathrm{LT}$, respectivamente) y pesados (945.7 y $661.5 \mathrm{~g}$ PT, respectivamente) respecto a los demás años. Aunque no 


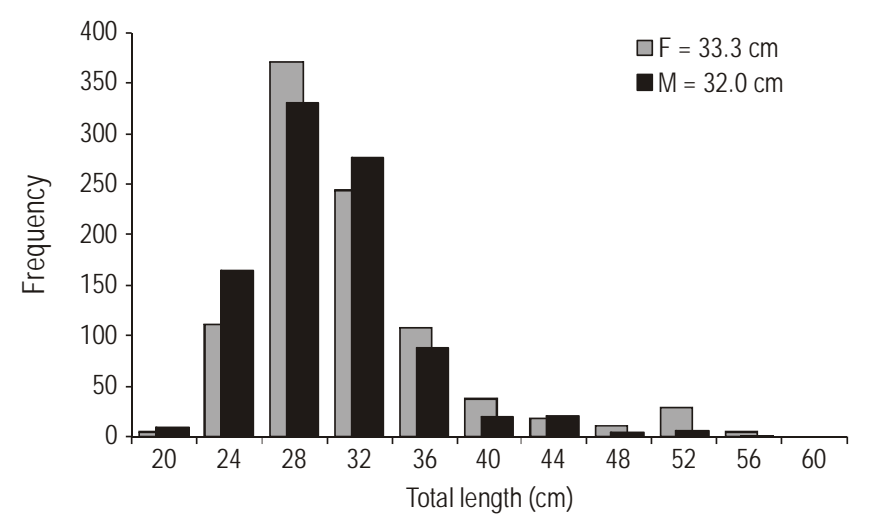

Figure 1. Distribution of length frequencies of female (F) and male (M) Lutjanus argentiventris from the Pacific coast of central Mexico. Mean total length.

Figura 1. Distribución de frecuencia de tallas de hembras (F) y machos (M) de Lutjanus argentiventris capturados en el Pacífico central mexicano. Longitud total promedio.

The sex ratio was calculated based on 1877 specimens, 945 females (50.3\%) and 932 males (49.7\%). The overall sex ratio $(\mathrm{F}: \mathrm{M})$ was 1:0.99, not significantly different from the expected 1:1 $\left(\chi^{2}=0.09, P=0.76\right)$. Significantly more females than males were observed in 1999 and 2008, whereas males dominated in 2000 and 2001 (table 1).

\section{Monthly variation of the macroscopic maturity stages}

The macroscopic maturity stages did not show a welldefined pattern to be able to precisely determine the breeding season. In females, the immature stage was observed throughout the study period, with frequency values above $30 \%$; the highest percentages of the developing stage were observed in August and September, and the highest percentage of the ripe stage was observed in September (fig. 2a). Males showed a similar percentage distribution of the maturity stages, with low percentages of immature individuals in April and July, and high percentages of the developing and ripe stages in April, August, and September (fig. 2b).

\section{Monthly variation of the gonadosomatic index (GSI) and condition factor (CF)}

The GSI values were highest from July to October for females and from July to September for males (fig. 3). The $\mathrm{CF}$ values were highest from May to July for females and from May to August for males (fig. 3). A correlation between GSI and CF was not observed in females but was observed in males.

\section{Monthly variation in mean oocyte diameter}

There were significant differences $(P<0.05$, ANOVA) in mean oocyte diameters between months (fig. 4). The fueron significativos $(P>0.05$, ANDEVA), los menores promedios de talla $(30.9 \mathrm{~cm} \mathrm{LT})$ y peso $(455.8 \mathrm{~g} \mathrm{PT})$ para las hembras se presentaron en 2001. Los machos fueron significativamente $(P<0.05$, ANDEVA) más pequeños, tanto en longitud (29.8 cm LT) como en peso (424.4 g PT), en 2000 (tabla 1).

La proporción sexual se calculó con base en 1877 ejemplares, 945 hembras (50.3\%) y 932 machos (49.7\%). La proporción sexual total fue de 1:0.99 (H:M) y no fue significativamente diferente de la esperada 1:1 $\left(\chi^{2}=0.09, P=\right.$ 0.76). Se observaron significativamente más hembras que machos en 1999 y 2008, mientras que los machos dominaron en 2000 y 2001 (tabla 1).

\section{Variación mensual de los estadios de madurez macroscópicos}

Los estadios de madurez macroscópicos no mostraron un patrón bien definido que permitiera delimitar de forma precisa el periodo reproductivo. En las hembras, el estadio inmaduro se presentó durante todo el periodo de estudio, con valores de frecuencia por arriba de $30 \%$; los mayores porcentajes del estadio en desarrollo se observaron en agosto y septiembre, y el máximo porcentaje del estadio maduro se observó en septiembre (fig. 2a). Los machos presentaron una tendencia semejante a la de las hembras en la distribución de porcentajes de estadios de madurez: se encontraron porcentajes bajos de individuos inmaduros en abril y julio, y porcentajes altos de individuos en estadio en desarrollo y maduro en abril, agosto y septiembre (fig. 2b).

\section{Variación mensual del índice gonadosomático (IGS) y del factor de condición (FC)}

Los valores máximos del IGS se observaron de julio a octubre para las hembras y de julio a septiembre para los machos (fig. 3). Los valores máximos del FC se observaron de mayo a julio para las hembras y de mayo a agosto para los machos (fig. 3). Para las hembras, no se observó una correlación entre el IGS y FC, pero sí en los machos.

\section{Variación mensual del diámetro promedio de los ovocitos}

Se encontraron diferencias significativas $(P<0.05$, ANDEVA) en los diámetros promedio de los ovocitos entre meses (fig. 4). La prueba de Newman-Keuls evidenció seis grupos, y en la mayoría de los meses se presentaron traslapos entre los grupos. Los diámetros promedio mayores se encontraron de julio a septiembre y los menores en noviembre y diciembre, y en el resto de los meses se presentaron los diámetros intermedios, observándose tres periodos (fig. 4). Se encontró una correlación positiva significativa temporal entre el IGS y el diámetro promedio de los ovocitos $\left(r_{\mathrm{s}}=\right.$ $0.91, P<0.001 ; n=12$ ). 


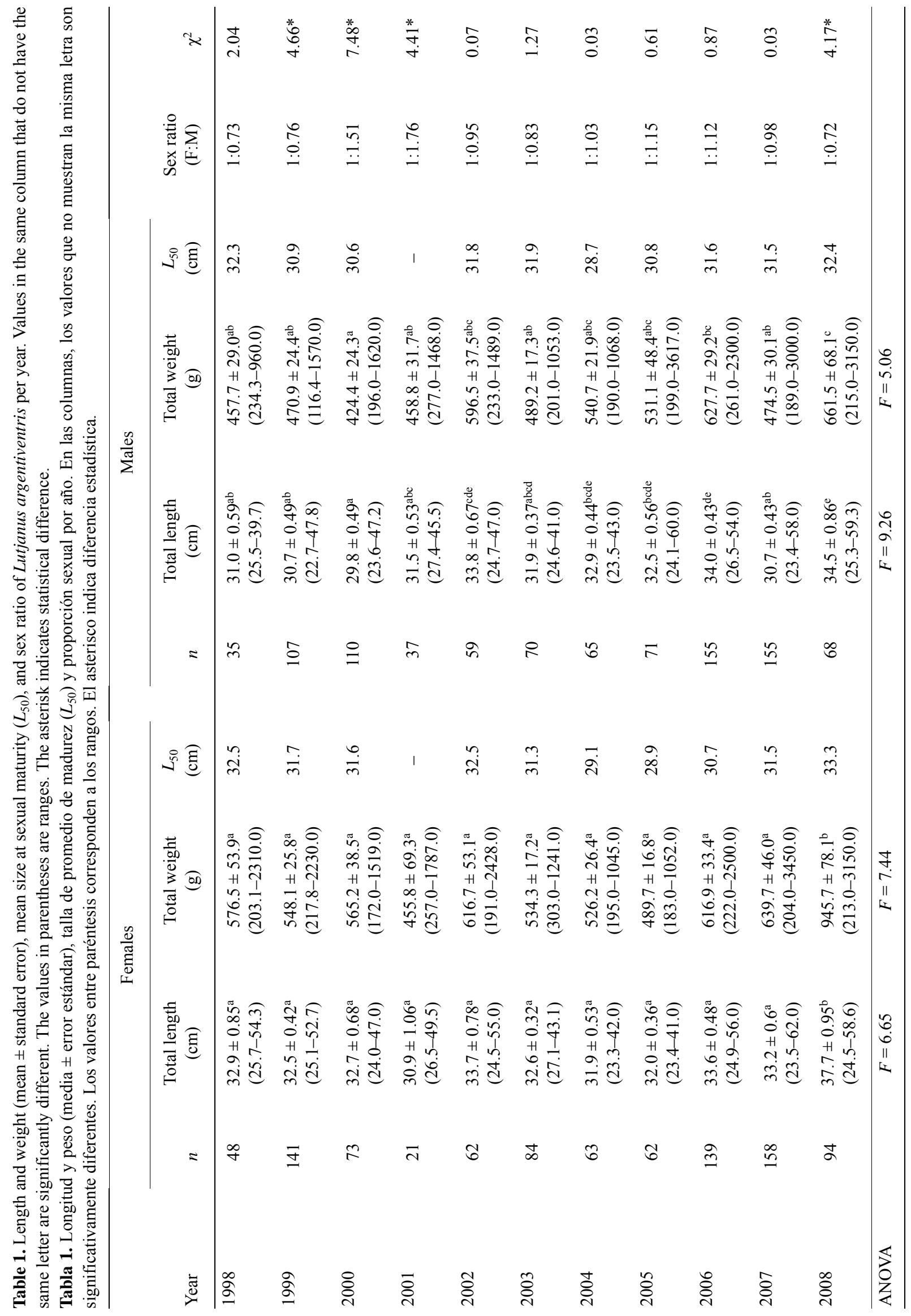




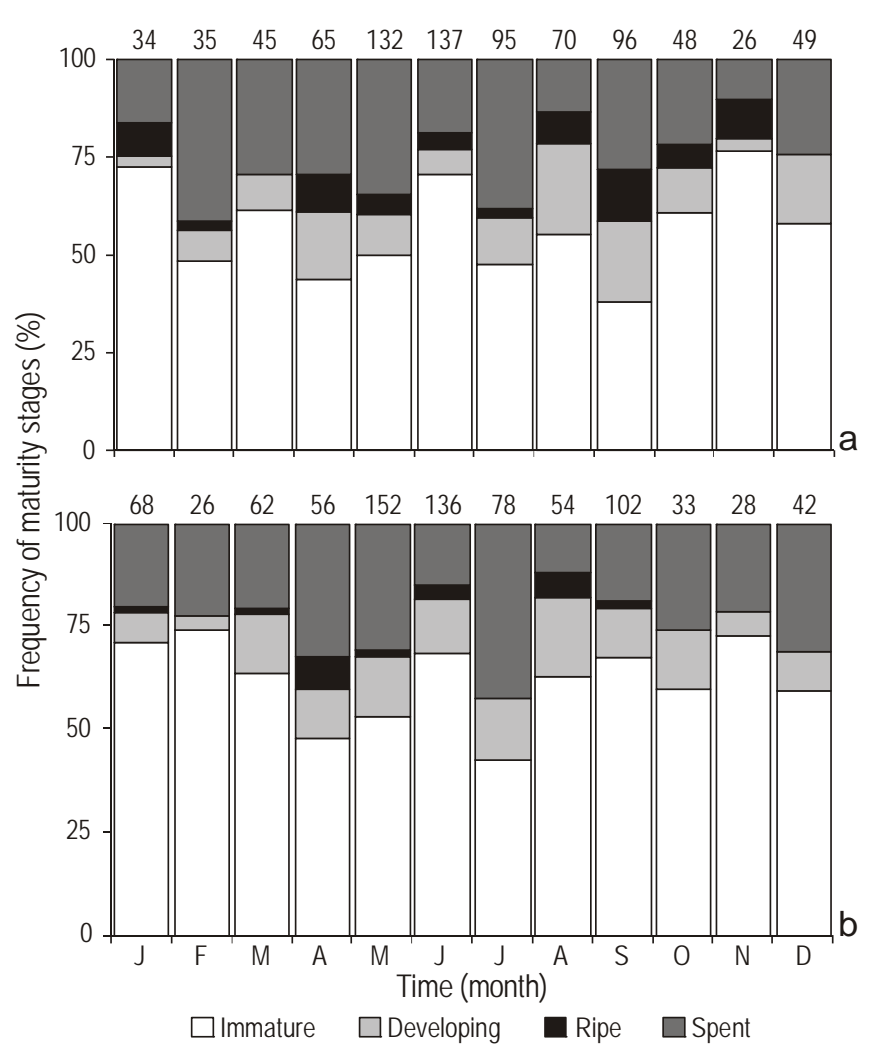

Figure 2. Frequency of gonad development stages in female (a) and male (b) Lutjanus argentiventris from the Pacific coast of central Mexico. Numbers on top indicate sample size.

Figura 2. Frecuencia de los estadios de madurez gonádica en hembras (a) y machos (b) de Lutjanus argentiventris capturados en el Pacífico central mexicano. Los números en la parte superior indican el tamaño de muestra.

Newman-Keuls test indicated six groups, and overlapping between groups occurred in most months. Highest mean diameters were found from July to September and lowest in November and December, and the rest of the months showed intermediate values, forming three periods (fig. 4). A significant positive temporal correlation was observed between GSI and mean oocyte diameter $\left(r_{\mathrm{s}}=0.91, P<0.001 ; n=12\right)$.

\section{Microscopic description of the gonads}

The ovary is encased by a tunic. Oocyte development inside the ovary is asynchronic. Seven stages of oocyte development were observed (fig. 5a, b). (1) Chromatinnucleolus stage: the oocyte has a mean diameter of $60.1 \mu \mathrm{m}$ $( \pm 0.55)$, little cytoplasm, and a large nucleus with nucleoli. (2) Perinucleolus stage: mean oocyte diameter of $80.9 \mu \mathrm{m}$ $( \pm 1.24)$ and nucleoli can be seen in the periphery of the nucleus. (3) Yolk vesicle stage: mean oocyte diameter of $169.1 \mu \mathrm{m}( \pm 2.45)$, oil droplets form in the cytoplasm, and yolk formation initiates. (4) Primary vitellogenesis stage: mean oocyte diameter of $248.32 \mu \mathrm{m}( \pm 3.02)$ and yolk

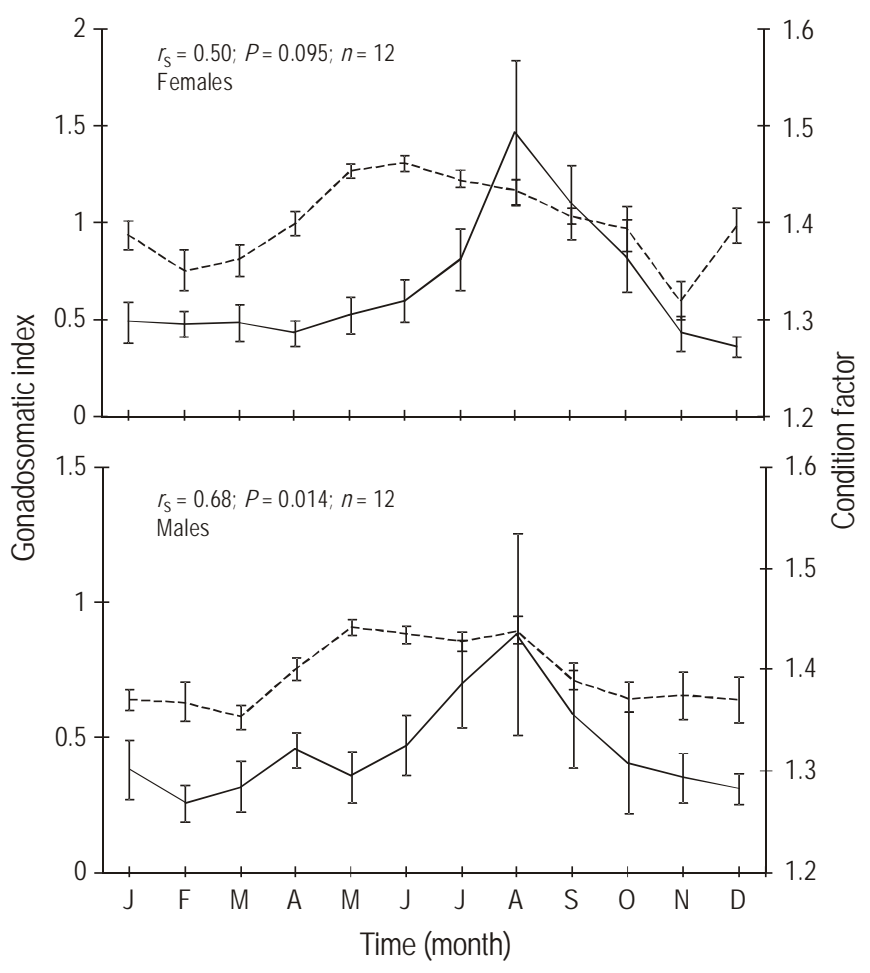

Figure 3. Monthly variation of the gonadosomatic index (solid line) and condition factor (dashed line) (mean \pm standard error) of female and male Lutjanus argentiventris from the Pacific coast of central Mexico.

Figura 3. Variación mensual del índice gonadosomático (línea continua) y del factor de condición (línea discontinua) (media \pm error estándar) de hembras y machos de Lutjanus argentiventris capturados en el Pacífico central mexicano.

\section{Descripción microscópica de las gónadas}

Los ovarios están envueltos por una túnica. El desarrollo de los ovocitos en el ovario es asincrónico. Se pudieron describir siete fases de desarrollo de los ovocitos (fig. 5a, b). (1) Fase cromatina nucléolo: el ovocito tiene un diámetro promedio de $60.1 \mu \mathrm{m}( \pm 0.55)$, poco citoplasma y un núcleo grande con nucléolos. (2) Fase en perinucléolo: el diámetro promedio del ovocito es de $80.9 \mu \mathrm{m}( \pm 1.24)$ y los nucléolos se observan en la periferia del núcleo. (3) Fase con vesículas vitelinas: el diámetro promedio del ovocito es de $169.1 \mu \mathrm{m}$ $( \pm 2.45)$, en el citoplasma se forman gotas de aceite e inicia la formación de vitelo. (4) Fase en vitelogénesis primaria: el diámetro promedio del ovocito es de $248.32 \mu \mathrm{m}( \pm 3.02)$ y se observan glóbulos de vitelo en el citoplasma. (5) Fase en vitelogénesis secundaria: el diámetro promedio del ovocito es de $322.76 \mu \mathrm{m}( \pm 3.77)$ y en todo el citoplasma se encuentran glóbulos de vitelo. (6) Fase en vitelogénesis terciaria: el diámetro promedio del ovocito es de $289.37 \mu \mathrm{m}( \pm 6.31)$, los glóbulos de vitelo se empiezan a fusionar y el núcleo se encuentra en proceso de migración hacia el polo animal. 


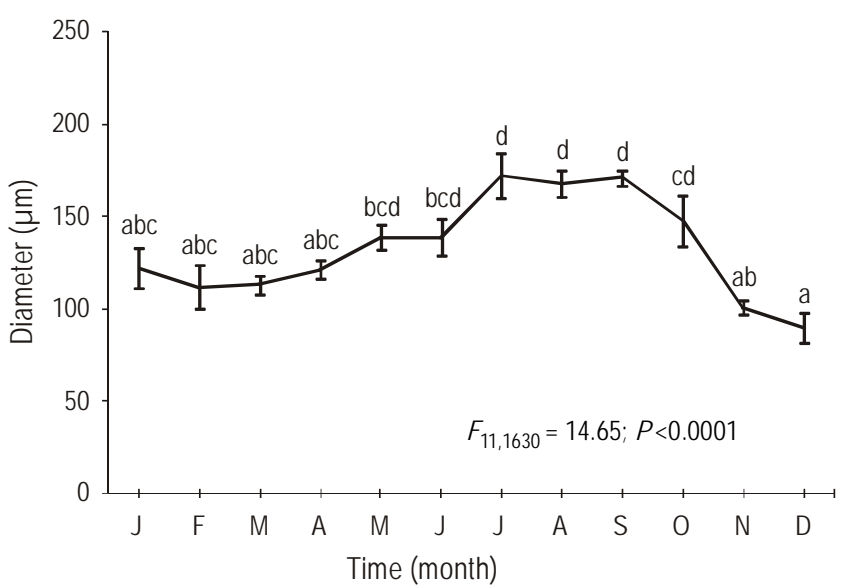

Figure 4. Monthly mean oocyte diameter of Lutjanus argentiventris (mean \pm standard error). Means that do not have the same letter are significantly different $(P<0.05)$.

Figura 4. Diámetro promedio mensual de los ovocitos de Lutjanus argentiventris (media \pm error estándar). Las medias que no presentan la misma letra son significativamente diferentes $(P<0.05)$.

globules can be seen in the cytoplasm. (5) Secondary vitellogenesis stage: mean oocyte diameter of $322.76 \mu \mathrm{m}( \pm 3.77)$ and yolk globules are visible throughout the cytoplasm. (6) Tertiary vitellogenesis stage: mean oocyte diameter of $289.37 \mu \mathrm{m}( \pm 6.31)$, the yolk globules begin to fuse, and the nucleus is in the process of migration towards the animal pole. (7) Ripe stage: mean oocyte diameter of $242.11 \mu \mathrm{m}$ $( \pm 5.33)$, the yolk is completely fused, and the nucleus is not visible.

The internal structure of the testis consists of lobules that are filled with cysts, which is where spermatogenesis occurs. Once formed, the spermatozoa move to the center the lobule and gather in the main duct to be expelled. This type of organization of the testis cells is referred to as lobular development (fig. 5c, d).

\section{Size at sexual maturity $\left(L_{50}\right)$}

The specimens were classified as immature or mature based on the macroscopic maturity stages. The smallest female and male with ripe gonads measured 26.0 and $24.6 \mathrm{~cm}$ $\mathrm{TL}$, respectively. The largest female and male with immature gonads measured 42.0 and $39.5 \mathrm{~cm}$ TL, respectively. Considering the cumulative relative frequencies, females between 26.0 and $33.3 \mathrm{~cm}$ TL represented $67.8 \%$ of all mature females, and males between 24.6 and $32.0 \mathrm{~cm}$ TL represented $57.3 \%$ of all mature males. The length at sexual maturity $\left(L_{50}\right)$ calculated based on data from all years was $31.6 \mathrm{~cm}$ TL for females and $31.4 \mathrm{~cm}$ TL for males (fig. 6). The $L_{50}$ values calculated per survey year ranged from $28.9 \mathrm{~cm} \mathrm{(2005)} \mathrm{to}$ $33.3 \mathrm{~cm} \mathrm{(2008)} \mathrm{for} \mathrm{females} \mathrm{and} \mathrm{from} 28.7 \mathrm{~cm} \mathrm{(2004)} \mathrm{to}$ $32.4 \mathrm{~cm}$ (2008) for males (table 1). Since the $L_{50}$ values for
(7) Fase madura: el diámetro promedio del ovocito es de $242.11 \mu \mathrm{m}( \pm 5.33)$, el vitelo está completamente fusionado y el núcleo no es visible.

El interior de los testículos se encuentra estructurado por lobulos que están formados por varios cistos, en los cuales se lleva a cabo la espermatogénesis. Una vez formados, los espermatozoides se dirigen al centro del lóbulo y se agrupan en el conducto principal para ser expulsados. A este tipo de organización que tienen las células del testículo se le denomina desarrollo lobular (fig. 5c, d).

\section{Talla de madurez sexual $\left(L_{50}\right)$}

Según los estadios de madurez macroscópicos, los ejemplares fueron clasificados como inmaduros o maduros. La hembra y el macho más pequeños con gónadas maduras midieron 26.0 y $24.6 \mathrm{~cm} \mathrm{LT}$, respectivamente. La hembra y el macho más grandes con gónadas inmaduras midieron $42.0 \mathrm{~cm}$ y $39.5 \mathrm{~cm} \mathrm{LT}$, respectivamente. Considerando las frecuencias relativas acumuladas, las hembras entre 26.0 y $33.3 \mathrm{~cm}$ LT representaron el $67.8 \%$ del total de hembras maduras, y los machos 24.6 y $32.0 \mathrm{~cm}$ LT representaron el $57.3 \%$ del total de machos maduros. La longitud de madurez sexual $\left(L_{50}\right)$ calculada utilizando los datos de todos los años fue de $31.6 \mathrm{~cm}$ LT para las hembras y $31.4 \mathrm{~cm}$ LT para los machos (fig. 6). La $L_{50}$ calculada para cada año de muestreo varió de $28.9 \mathrm{~cm}$ (2005) a $33.3 \mathrm{~cm}$ (2008) para las hembras y de $28.7 \mathrm{~cm}$ (2004) a $32.4 \mathrm{~cm}$ (2008) para los machos (tabla 1). Debido a que la $L_{50}$ fue semejante entre sexos en cada año, se combinaron los datos de ambos sexos. El análisis de regresión no presentó alguna tendencia estadísticamente significativa $\left(F_{1,8}=0.129 ; P<0.728\right)$ hacia el aumento o disminución de la talla de madurez.

\section{DISCUSIÓN}

El intervalo de longitud de $L$. argentiventris registrado en el presente trabajo (21.5 a $62.0 \mathrm{~cm} \mathrm{LT}$ ) es semejante al presentado por Piñón et al. (2009) para el golfo de California (10.8 a $59.0 \mathrm{~cm} \mathrm{LT),} \mathrm{Rojas} \mathrm{et} \mathrm{al.} \mathrm{(2004)} \mathrm{para} \mathrm{Colombia}$ (20.0 a $70.1 \mathrm{~cm}$ LT) y Cruz-Romero et al. (1996) para Colima (24.0 a $56.5 \mathrm{~cm}$ de longitud estándar); sin embargo, es más amplio al presentado por Rojo-Vázquez et al. (1999) para Jalisco (19.0 a $34.5 \mathrm{~cm}$ LT). Esta discrepancia puede atribuirse a que Rojo-Vázquez et al. (1999) utilizaron redes agalleras con una la luz de malla menor. Los promedios de talla y de peso aquí presentados $(32.7 \pm 0.13 \mathrm{~cm}$ LT y $567.3 \pm$ $9.14 \mathrm{~g}$ PT) fueron mayores que los registrados por Piñón et al. (2009) para el golfo de California (24.4 $\pm 7.7 \mathrm{~cm} \mathrm{LT} \mathrm{y}$ $280.5 \pm 368.3 \mathrm{~g} \mathrm{PT}$ ), posiblemente debido a un gran número de organismos juveniles en la muestra de estos últimos autores. La talla promedio de pargo alazán encontrada por Rojas et al. (2004) en un parque natural en Colombia es superior $(48.2 \mathrm{~cm})$ a la encontrada en el presente trabajo; esto quizá es el resultado del gran número de organismos muestreados en 

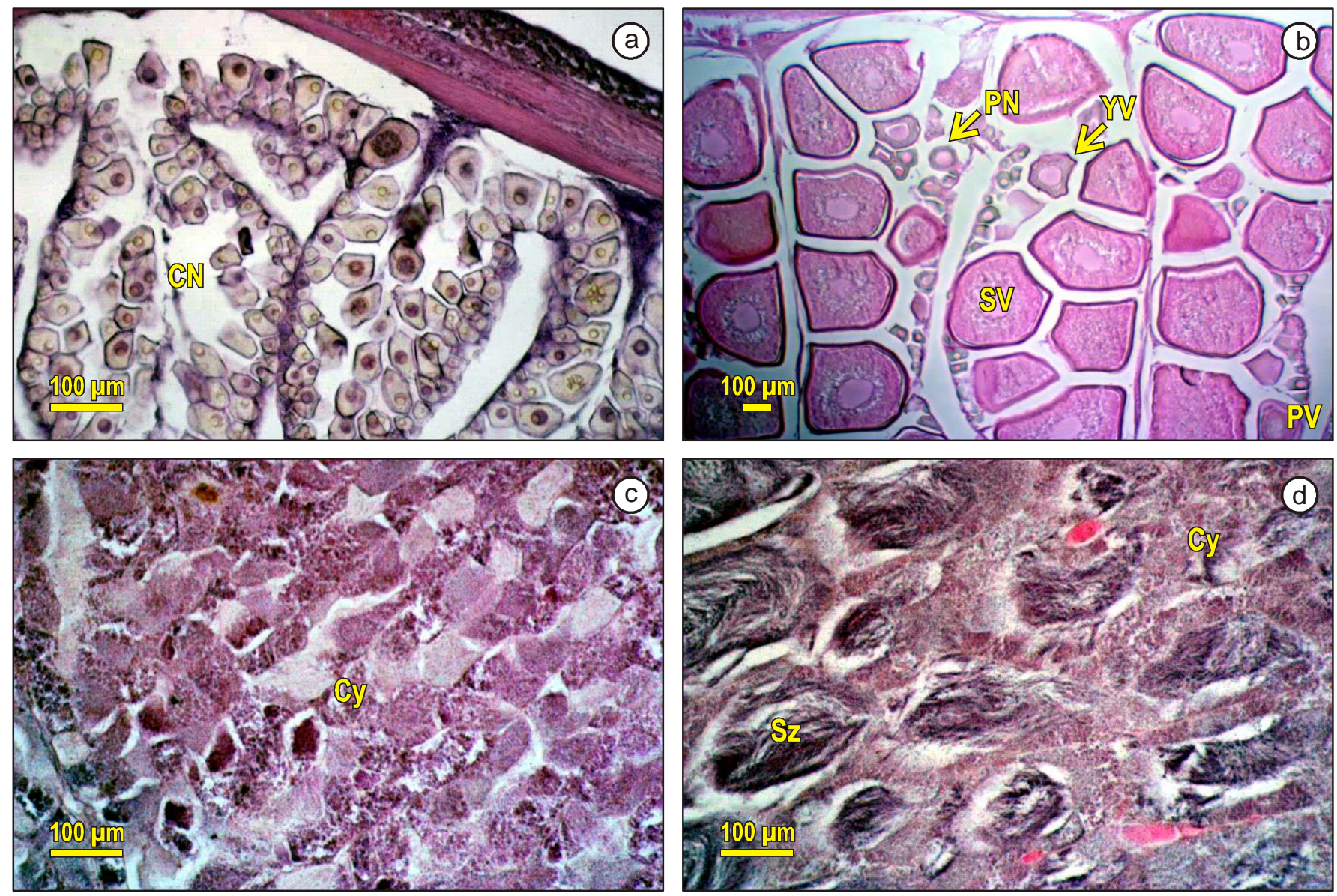

Figure 5. Cross section $(10 \times)$ of the ovary $(\mathbf{a}, \mathbf{b})$ and testis $(\mathbf{c}, \mathbf{d})$ of Lutjanus argentiventris. (a) Immature ovary with chromatin-nucleolus (CN) oocytes. (b) Developing ovary with oocytes at different stages of development: perinucleolus (PN), yolk vesicle (YV), primary vitellogenesis (PV), and secondary vitellogenesis (SV). (c) Immature testis with cysts (Cy) at different stages of development. (d) Ripe testis with spermatozoa (Sz) in the center of the lobules and cysts (Cy) in the periphery.

Figura 5. Corte transversal $(10 \times)$ del ovario (a, b) y testículo (c, d) de Lutjanus argentiventris. (a) Ovario inmaduro con ovocitos en cromatina nucléolo (CN). (b) Ovario en desarrollo con ovocitos en diferentes fases de desarrollo: perinucléolo (PN), vesículas vitelinas (YV), vitelogénesis primaria (PV) y vitelogénesis secundaria (SV). (c) Testículo inmaduro con cistos (Cy) en diferentes fases de desarrollo. (d) Testículo en reproducción con espermatozoides (Sz) en el centro de los lóbulos y cistos (Cy) en la periferia.

both sexes were similar, the data were pooled. The regression analysis did not show any statistically significant increasing or decreasing trend $\left(F_{1,8}=0.129 ; P<0.728\right)$ in size at maturity.

\section{DISCUSSION}

The size range for L. argentiventris presented herein $(21.5$ to $62.0 \mathrm{~cm} \mathrm{TL}$ ) is similar to that found by Piñón et al. (2009) in the Gulf of California (10.8 to $59.0 \mathrm{~cm} \mathrm{TL}$ ), by Rojas et al. (2004) in Colombia (20.0 to $70.1 \mathrm{~cm} \mathrm{TL}$ ), and by CruzRomero et al. (1996) in Colima (24.0 to $56.5 \mathrm{~cm}$ standard length); however, it is broader than that found by RojoVázquez et al. (1999) in Jalisco (19.0 to $34.5 \mathrm{~cm} \mathrm{TL}$ ). The reason for this discrepancy could be that Rojo-Vázquez et al. (1999) used gillnets with a smaller mesh size. Our mean size and weight values $(32.7 \pm 0.13 \mathrm{~cm} \mathrm{TL}$ and $567.3 \pm 9.14 \mathrm{~g}$ tallas de 40.0 a $55.0 \mathrm{~cm}$, tallas que son poco capturadas en la pesca comercial en nuestra región.

Aunque en cuatro de los diez años se encontraron diferencias significativas en la proporción de sexos, éstas no afectaron la proporción total. Torres (1996), Cruz-Romero et al. (1996) y Rojas et al. (2004) encontraron un mayor número de machos que de hembras. En el presente trabajo y en el de Piñón et al. (2009), se capturaron más hembras que machos. Piñón et al. (2009) observaron un mayor número de machos que de hembras en los periodos reproductivos, y en el presente trabajo se encontraron más hembras que machos en julio. Se ha sugerido que las diferencias en la proporción de sexos pueden estar ligadas a la diferencia en la supervivencia de los sexos, la distribución y preferencia del hábitat, la profundidad, la distribución por tallas (juveniles/adultos), y los aspectos alimenticios y reproductivos (Nikolsky 1963, CruzRomero et al. 1996, Rojas et al. 2004, Piñón et al. 2009). 
TW) are higher than those reported by Piñón et al. (2009) for the Gulf of California $(24.4 \pm 7.7 \mathrm{~cm}$ TL and $280.5 \pm 368.3 \mathrm{~g}$ TW), most likely because of the large number of juvenile organisms in the sample of the latter study. The mean size obtained by Rojas et al. (2004) for specimens from a national park in Colombia $(48.2 \mathrm{~cm})$ is higher than that recorded in the present study, probably as a result of the large number of organisms examined measuring between 40.0 and $55.0 \mathrm{~cm}$, sizes that are rarely caught in the commercial fishery of our region.

Though significant differences in sex ratio were found in four of the ten years, the overall sex ratio was not affected. Torres (1996), Cruz-Romero et al. (1996), and Rojas et al. (2004) recorded a greater number of males than females. In the present study and in that of Piñon et al. (2009), more females than males were captured. During the breeding season, Piñón et al. (2009) observed a greater number of males than females, whereas in July we found more females than males. It has been suggested that differences in sex ratios can be attributed to differences in survival between males and females, habitat preference and distribution, depth, size distribution (juveniles/adults), and feeding and breeding aspects (Nikolsky 1963, Cruz-Romero et al. 1996, Rojas et al. 2004, Piñón et al. 2009).

The breeding season reported for $L$. argentiventris along the Pacific coast of Mexico varies slightly. Piñón et al. (2009) observed that in the Gulf of California, the species reproduces in summer and winter. Muhlia-Melo et al. (2003) indicate that in Baja California Sur, organisms in captivity spawn from July to September, with maximum reproductive activity in August. Cruz-Romero et al. (1996) found that off Colima the breeding season occurs from April to May and from September to November. Comparing these results with those obtained in the present study, we can conclude that off the coast of Jalisco, L. argentiventris reproduces in summer, from July to September, with maximum reproductive activity in August.

The CF is a variable that is used to indicate the physiological condition of fish. A negative correlation between CF and GSI, attributed to energy allocation to gonad maturation, has been reported for some species (Maddock and Burton 1999, Arellano-Martínez and Ceballos-Vázquez 2001, González and Oyarzún 2002). Like Sánchez-Cárdenas et al. (2007), in the present study we did not find a negative correlation between GSI and CF; however, in both male and female yellow snapper, the $\mathrm{CF}$ showed high values before the maximum values of the GSI, and after reaching the highest values, both the GSI and CF decreased, resulting in a positive correlation in females.

Asynchronous spawning (partial and fractional) has been reported for Scomberomorus sierra (Lucano Ramírez et al. 2011b), Prionotus ruscarius (Lucano-Ramírez et al. 2005), Pseudupeneus grandisquamis (Lucano-Ramírez et al. 2006), and Diodon holocanthus (Lucano-Ramírez et al. 2011a), species that are distributed in tropical and subtropical

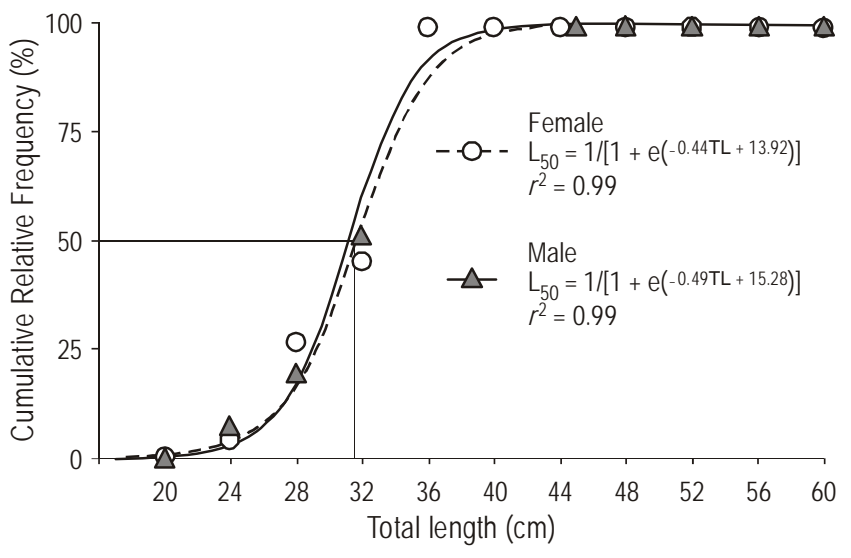

Figure 6. Cumulative relative frequency of mature male and female Lutjanus argentiventris from the Pacific coast of central Mexico. Values fitted to the logistic equation. TL is the total length.

Figura 6. Frecuencia relativa acumulada de hembras y machos maduros de Lutjanus argentiventris capturados en el Pacífico central mexicano. Valores ajustados a la ecuación logística. TL es la longitud total.

El periodo reproductivo que se ha documentado para L. argentiventris en el litoral del Pacífico mexicano ha presentado ligeras variaciones. Piñón et al. (2009) mencionaron que en el golfo de California, la especie se reproduce en verano e invierno. Muhlia-Melo et al. (2003), al examinar organismos en cautiverio en Baja California Sur, señalaron que la especie desovó de julio a septiembre, con un máximo de actividad reproductiva en agosto. Cruz-Romero et al. (1996) determinaron que el periodo reproductivo en Colima sucede en abril-mayo y septiembre-noviembre. Si se comparan estos resultados con los obtenidos en el presente trabajo, se puede concluir que en las costas de Jalisco, L. argentiventris se reproduce en verano, de julio a septiembre, con un máximo de actividad reproductiva en agosto.

El FC es una variable que se utiliza para indicar la condición fisiológica de los peces. Para algunas especies, se ha encontrado que el FC presenta una correlación negativa con el IGS debido a la energía que se canaliza para la maduración de las gónadas (Maddock y Burton 1999, ArellanoMartínez y Ceballos-Vázquez 2001, González y Oyarzún 2002). Al igual que Sánchez-Cárdenas et al. (2007), en el presente trabajo no se encontró una correlación negativa entre el IGS y FC; sin embargo, tanto en hembras como en machos de pargo alazán, el FC presentó valores altos antes que los puntos máximos del IGS, y después de alcanzar valores máximos, el IGS y el FC disminuyeron, lo que resultó en una correlación positiva en las hembras.

El desove asincrónico (parcial o fraccionado) se presenta en especies como Scomberomorus sierra (Lucano Ramírez et al. 2011b), Prionotus ruscarius (Lucano-Ramírez et al. 2005), Pseudupeneus grandisquamis (Lucano-Ramírez et al. 2006) y Diodon holocanthus (Lucano-Ramírez et al. 2011a), 
regions. Species from temperate regions, however, have a short breeding season, present synchronous gonadal development, and are total spawners (Nikolsky 1963, GranadosLorencio 1996). Similar to Piñón et al. (2009), we observed asynchronous oocyte development in L. argentiventris. Fish species with asynchronous ovarian development generally spawn on several occasions in a relatively long breeding season (Nagahama et al. 1995, Maack and George 1999), and these ovaries contain oocytes of all stages of development.

In $L$. argentiventris, we identified seven stages of oocyte development, representing most of oogenesis. Other studies have also identified from five to seven stages in other species caught off the coast of Jalisco (Lucano-Ramírez et al. 2001a, 2005, 2006, 2011a，b). Sánchez-Cárdenas et al. (2011) described seven stages of oocyte development in Sphoeroides annulatus, similar to those observed in the present study. González et al. (1979) reported five stages for Lutjanus griseus: stages I, II, III, and IV (immature oocytes) were observed in wild-caught organisms, and stage V (mature oocytes) was observed in females treated with the hormone human chorionic gonadotropin. Piñón et al. (2009) described five stages in L. argentiventris but did not mention if fully mature oocytes were observed. It is difficult to observe mature oocytes in wild-caught fish because the process of oocyte hydration occurs in a few hours (González et al. 1979, Muhlia-Melo et al. 2003); nonetheless, in the present study, it was possible to observe ripe oocytes.

There are two types of testicular development: lobular and tubular. Lobular development has been observed in L. peru (Lucano-Ramírez et al. 2001a), D. holocanthus (Lucano-Ramírez et al. 2011a), and S. sierra (LucanoRamírez et al. 2011b) caught off the southern coast of Jalisco. Like Sánchez-Cárdenas et al. (2011), we observed a main duct, located in the central part of each testis. Its function is to collect and store the sperm that will be expelled.

The logistic model is one of the most widely used methods to estimate size at sexual maturity (Schmidt et al. 1993, Mackie et al. 2005, Aguirre-Villaseñor et al. 2006, Sadeghi et al. 2009, Lucano-Ramírez et al. 2011b). Piñón et al. (2009) and Rojas et al. (2004) used this model to estimate the $L_{50}$ of $L$. argentiventris. Piñón et al. (2009) obtained a similar value $(32.6 \mathrm{~cm} \mathrm{TL})$ to that reported herein; however, Rojas et al. (2004), obtained a higher value $(51.5 \mathrm{~cm} \mathrm{TL})$. The specimens examined by Rojas et al. (2004) came from a national park in Colombia and not from commercial landings and this may explain their larger size and high $L_{50}$ values. Cruz-Romero et al. (1996) reported that the minimum size at maturity of female snapper caught off Colima ranged from 24.8 to $25.9 \mathrm{~cm}$ (data transformed to TL). Piñón et al. (2009) reported that the smallest size of a mature female yellow snapper from the Gulf of California was $22.8 \mathrm{~cm}$ TL. In the present study (southern coast of Jalisco), the minimum size of a mature female was $26 \mathrm{~cm} \mathrm{TL}$ and of a mature male, $24.6 \mathrm{~cm} \mathrm{TL}$. All these minimum sizes at maturity coincide, only the minimum size $(31.0 \mathrm{~cm} \mathrm{TL})$ reported by Rojas et al. que se distribuyen en regiones tropicales y subtropicales. En contraste, los peces de las regiones templadas tienen un periodo reproductivo corto, el desarrollo de la gónada es sincrónico y el desove es total (Nikolsky 1963, GranadosLorencio 1996). Tanto el presente trabajo como el de Piñón et al. (2009) documentan el desarrollo asincrónico de los ovocitos en L. argentiventris. Los peces cuyos ovarios presentan desarrollo asincrónico, por lo general, desovan en varias ocasiones en una temporada reproductiva relativamente prolongada (Nagahama et al. 1995, Maack y George 1999), y estos ovarios contienen ovocitos en diferentes fases de desarrollo.

En el presente trabajo se identificaron siete fases de desarrollo de ovocitos de L. argentiventris, las cuales representan gran parte de la ovogénesis. Otros trabajos realizados en la región han registrado de cinco a siete fases para otras especies capturadas frente a la costa de Jalisco (Lucano-Ramírez et al. 2001a, 2005, 2006, 2011a, b). Sánchez-Cárdenas et al. (2011) encontraron siete fases de desarrollo para los ovocitos de Sphoeroides annulatus; estas fases son semejantes a las observadas en este estudio. González et al. (1979) describieron cinco fases de desarrollo para Lutjanus griseus, y mencionaron que las fases I, II, III y IV (no maduras) fueron observadas en organismos capturados en el medio natural, mientras que la fase V (ovocitos maduros) fue observada en hembras tratadas con la hormona gonadotropina coriónica humana. Piñón et al. (2009) describieron cinco fases de desarrollo en L. argentiventris, pero no mencionaron si encontraron ovocitos completamente maduros. Es difícil observar ovocitos maduros en organismos capturados en el medio natural, pues la hidratación del ovocito es un proceso que ocurre en pocas horas (González et al. 1979, Muhlia-Melo et al. 2003); no obstante, en este estudio fue posible observar ovocitos maduros.

Existen dos tipos de desarrollo testicular: el lobular y el tubular. El desarrollo testicular lobular se ha observado en L. peru (Lucano-Ramírez et al. 2001a), D. holocanthus (Lucano-Ramírez et al. 2011a) y S. sierra (Lucano-Ramírez et al. 2011b) en la costa sur de Jalisco. Al igual que SánchezCárdenas et al. (2011), observamos un conducto principal que recorre la parte central de cada testículo, y función es recoger y guardar los espermatozoides que son liberados al momento de la expulsión.

El modelo logístico es uno de los métodos más utilizados para estimar la talla de madurez sexual (Schmidt et al. 1993, Mackie et al. 2005, Aguirre-Villaseñor et al. 2006, Sadeghi et al. 2009, Lucano-Ramírez et al. 2011b). Piñón et al. (2009) y Rojas et al. (2004) utilizaron este modelo para estimar la $L_{50}$ de $L$. argentiventris. El valor estimado por Piñón et al. (2009) (32.6 cm LT) fue semejante al obtenido en esta investigación, mientras que el valor estimado por Rojas et al. (2004) (51.5 cm LT) resultó mayor. Los organismos examinados por Rojas et al. (2004) no provinieron de la captura comercial, sino de un parque nacional en Colombia, y quizá esto explica el valor alto de la $L_{50}$ y las mayores tallas de los organismos que recolectaron. Cruz-Romero et al. 
(2004) differs. On the other hand, the mean size of all the organisms sampled in this study was slightly higher than the $L_{50}$, which suggests that a little more than $50 \%$ of the organisms captured had reproduced at least once.

\section{ACKNOWLEDGMENTS}

This study was funded by the University of Guadalajara. The authors thank Daniel Kosonoy, Gerardo Kosonoy, and Manuel Díaz for their help with the fishing activities, the cooperatives "Rivera Melaque" and "Punta Farallón" for use of their installations, and the students who participated in the surveys.

\section{English translation by Christine Harris.}

\section{REFERENCES}

Aguirre-Villaseñor H, Morales-Bojórquez E, Morán-Angulo RE, Madrid-Vera J, Valdez-Pineda MC. 2006. Indicadores biológicos de la pesquería de sierra (Scomberomorus sierra) al sur del golfo de California, México. Cienc. Mar. 32: 471-484.

Allen GR, Robertson DR. 1998. Peces del Pacífico Oriental Tropical. Crawford House Press, 334 pp.

Amezcua-Linares F. 1996. Peces Demersales de la Plataforma Continental del Pacífico Central de México. Instituto de Ciencias del Mar y Limnología, Universidad Nacional Autónoma de México, y Comisión Nacional para el Conocimiento y Uso de la Biodiversidad, $184 \mathrm{pp}$.

Arellano-Martínez M, Ceballos-Vázquez BP. 2001. Reproductive activity and condition index of Holocanthus passer (Teleostei: Pomacanthidae) in the Gulf of California, Mexico. Rev. Biol. Trop. 49: 939-943.

Cerisola H. 1984. Cambios estructurales del folículo ovárico durante su maduración en el pejesapo Sicyases sanguineus, Muller y Troschel 1843 (Gobiescocidae: Teleostei). Rev. Biol. Mar. (Valparaíso) 20: 1-21.

Cruz-Romero M, Chávez EA, Espino E, García A. 1996. Assessment of a snapper complex (Lutjanus spp.) of the eastern tropical Pacific. In: Arreguín-Sánchez F, Munro JL, Balgos MC, Pauly D (eds.), Biology, Fisheries and Culture of Tropical Groupers and Snappers. ICLARM Conf. Proc. 48, Manila, Philippines, pp. 324-330.

Espino-Barr E, Cabral-Solís EG, García-Boa A, Puente-Gómez M. 2004. Especies Marinas con Valor Comercial de la Costa de Jalisco, México. CRIP, Manzanillo, México, 145 pp.

Everson AR, Williams HA, Ito BM. 1989. Maturation and reproduction in two Hawaiian eteline snappers, Uku, Aprion virescens, and Onaga, Etelis coruscans. Fish. Bull. 87: 877-888.

González E, Damas T, Millares N, Borrero M. 1979. Desove inducido en el caballerote (Lutjanus griseus Linné 1758) en condiciones de laboratorio. Rev. Cub. Inv. Pesq. 4: 43-63.

González P, Oyarzún C. 2002. Variabilidad de índices biológicos en Pinguipes chilensis Valenciennes 1833 (Perciformes, Pinguipedidae): ¿Están realmente correlacionados? Gayana 66: 249-253.

Granados-Lorencio C. 1996. Ecología de Peces. Secretariado de Publicaciones, Universidad de Sevilla, 353 pp.

Hyder M. 1969. Histological studies on the testis of Tilapia leucosticta and other species of the genus Tilapia (Pisces: Teleostei). Trans. Am. Microsc. Soc. 88: 211-231.
(1996) registraron que el intervalo de la talla mínima de madurez de hembras de L. argentiventris en Colima fue de 24.8 a $25.9 \mathrm{~cm}$ (datos transformados a LT). Piñón et al. (2009) registraron que la hembra madura más pequeña de L. argentiventris capturada en el golfo de California midió $22.8 \mathrm{~cm}$ LT. En nuestro trabajo (costa sur de Jalisco), la talla mínima de un organismo maduro fue de $26 \mathrm{~cm}$ LT para las hembras y $24.6 \mathrm{~cm}$ LT para los machos. Todos estos resultados de talla mínima de madurez son cercanos, sólo difiere la talla mínima (31.0 cm LT) registrada por Rojas et al. (2004). Por otro lado, la talla promedio del total de organismos muestreados en este trabajo fue ligeramente superior a la $L_{50}$, lo cual sugiere que poco más del $50 \%$ de los organismos capturados ya se habían reproducido por lo menos una vez.

\section{Agradecimientos}

Este estudio fue financiado por la Universidad de Guadalajara. Los autores agradecen a Daniel Kosonoy, Gerardo Kosonoy y Manuel Díaz su ayuda en la realización de las actividades de pesca, a las cooperativas "Rivera Melaque" y "Punta Farallón" el uso de sus instalaciones y a los estudiantes que colaboraron en los muestreos.

Lucano-Ramírez G, Villagrán-Santa Cruz M, Ruiz-Ramírez S. 2001a. Cambios estructurales en las gónadas de Lutjanus peru (Pisces: Lutjanidae), en la costa sur de Jalisco, México. Bol. Centro Invest. Biol. 35: 295-316.

Lucano-Ramírez G, Villagrán-Santa Cruz M, Ruiz-Ramírez S, López-Trujillo MT. 2001b. Histology of the oocytes of Lutjanus peru (Nichols and Murphy 1922) (Pisces: Lutjanidae). Cienc. Mar. 27: 335-349.

Lucano-Ramírez G, Ruiz-Ramírez S, Rojo-Vázquez JA. 2005. Biología reproductiva de Prionotus ruscarius (Pisces: Triglidae) en las costas de Jalisco y Colima, México. Rev. Digital Univ. 6: 1067-1079.

Lucano-Ramírez G, Ruiz-Ramírez S, Rojo-Vázquez JA. 2006. Composición por tallas y ciclo reproductivo de Pseudupeneus grandisquamis (Pisces: Mullidae) en el Pacífico central mexicano. Rev. Biol. Trop. 54: 195-207.

Lucano-Ramírez G, Peña-Pérez E, Ruiz-Ramírez S, Rojo-Vázquez JA, González-Sansón G. 2011a. Aspectos reproductivos del pez erizo, Diodon holocanthus (Pisces: Diodontidae) en la plataforma continental del Pacífico central mexicano. Rev. Biol. Trop. 59: 217-232.

Lucano-Ramírez G, Ruiz-Ramírez S, Palomera-Sánchez FI, González-Sansón G. 2011b. Biología reproductiva de la sierra Scomberomorus sierra (Pisces, Scombridae) en el Pacífico central mexicano. Cienc. Mar. 37: 249-260.

Maack G, George MR. 1999. Contributions to the reproductive biology of Encrasicholina punctifer Fowler 1938 (Engraulidae) from West Sumatra, Indonesia. Fish. Res. 44: 113-120.

Mackie MC, Lewis PD, Gaughan DJ, Newman SJ. 2005. Variability in spawning frequency and reproductive development of the narrow-barred Spanish mackerel (Scomberomorus commerson) along the west coast of Australia. Fish. Bull. 103: 344-354.

Maddock DM, Burton MPM. 1999. Gross and histological observations of ovarian development and related condition changes in American plaice. J. Fish Biol. 53: 928-944. 
Muhlia-Melo A, Guerrero-Tortolero DA, Pérez-Urbiola JC, Campos-Ramos R. 2003. Results of spontaneous spawning of yellow snapper (Lutjanus argentiventris Peters 1869) reared in inland ponds in La Paz, Baja California Sur, Mexico. Fish Physiol. Biochem. 28: 511-512.

Nagahama Y, Yoshikuni M, Yamashita T, Tokumoto M, Katsu Y. 1995. Regulation of oocyte growth and maturation in fish. Curr. Top. Dev. Biol. 30: 103-145.

Nikolsky G. 1963. The Ecology of Fishes. Academic Press, London.

Piñón A, Amezcua F, Duncan N. 2009. Reproductive cycle of female yellow snapper Lutjanus argentiventris (Pisces, Actinopterygii, Lutjanidae) in the SW Gulf of California: Gonadic stages, spawning seasonality and length at sexual maturity. J. Appl. Ichthyol. 25: 18-25.

Rojas PA, Gutiérrez FC, Puentes V. 2004. Aspectos de la biología y dinámica poblacional del pargo coliamarillo Lutjanus argentiventris en el Parque Nacional Natural Gorgona, Colombia. Invest. Mar. (Valparaíso) 32: 23-36.

Rojo-Vázquez JA, Arreguín-Sánchez F, Godínez-Domínguez E, Ramírez-Rodríguez M. 1999. Gillnet selectivity for the spotted rose snapper (Lutjanus guttatus) and the amarillo snapper (Lutjanus argentiventris) in Navidad Bay, Jalisco, Mexico. Cienc. Mar. 25: 145-152.

Rojo-Vázquez JA, Quiñones-Velázquez C, Echavarría-Heras HA, Lucano-Ramírez G, Godínez-Domínguez E, Ruiz-Ramírez S, Galván-Piña VH, Sosa-Nishizaki O. 2008. The fish species composition and variation of catch from the small-scale gillnet fishery before, during and after the 1997-1998 ENSO event, central Mexican Pacific. Rev. Biol. Trop. 56: 133-152.

Sadeghi MS, Kaymaram F, Jamili S, Fatemi MR, Mortazavi MS. 2009. Patterns of reproduction and spawning of Scomberomorus commerson in the coastal waters of Iran. J. Fish. Aquat. Sci. 4: $32-40$.
Sánchez-Cárdenas R, Ceballos-Vázquez BP, Arellano-Martínez M, Valdez-Pineda MC, Morán-Angulo RE. 2007. Reproductive aspects of Sphoeroides annulatus (Jenyns 1842) (Tetraodontiformes, Tetraodontidae) inhabiting the Mazatlan coast, Sinaloa, Mexico. Rev. Biol. Mar. Oceanogr. 42: 385-392.

Sánchez-Cárdenas R, Arellano-Martínez M, Valdez-Pineda MC, Morán-Angulo RE, Ceballos-Vázquez BP. 2011. Reproductive cycle and sexual maturity of Sphoeroides annulatus (Jenyns 1842) (Tetraodontiformes, Tetraodontidae) from the coast of Mazatlan, Sinaloa, Mexico. J. Appl. Ichthyol. 27: 1190-1196.

Schmidt DJ, Collins MR, Wyanski DM. 1993. Age, growth, maturity, and spawning of Spanish mackerel, Scomberomorus maculatus (Mitchill), from the Atlantic coast of the southeastern United States. Fish. Bull. 91: 526-533.

Torres CA. 1996. Aspectos biológico-pesqueros del pargo planero Lutjanus argentiventris (Peters 1869) y reconocimiento sobre la pesca artesanal en el municipio de bahía Solano (ChocóColombia). Informe Técnico, Instituto Nacional de Pesca y Acuicultura INPA, Colombia, 16 pp.

Vitale F, Svedäng H, Cardinale M. 2006. Histological analysis invalidates macroscopically determined maturity ogives of the Kattegat cod (Gadus morhua) and suggests new proxies for estimating maturity status of individual fish. J. Mar. Sci. 63: 485-492.

West G. 1990. Methods of assessing ovarian development in fishes: a review. Aust. J. Mar. Fresh. Res. 41: 199-222 http://dx.doi.org/10.1071/MF9900199

Yamamoto K, Yamazaki M. 1961. Rhythm of development in the oocyte of the gold-fish, Carassius auratus. Bull. Fac. Fish. Hokkaido Univ. 12: 93-114.

Zar JH. 2010. Bioestatistical Analysis. 5th ed. Prentice Hall, New Jersey, 994 pp.

Received June 2013, accepted January 2014. 